\title{
A Lane Marking Extraction Approach based on Random Finite Set Statistics
}

\author{
Feihu Zhang, Hauke Stähle, Chao Chen, Christian Buckl and Alois Knoll
}

\begin{abstract}
Within the past few years, lane detection technology has become of high interest in the field of intelligent vehicles; however, robustness is still an issue. The challenge is to extract the lane markings effectively from the complex urban environment. In this paper, we present a novel approach based on Random Finite Set Statistics for estimating the position of lane markings. We rely on Probability Hypothesis Density (PHD) filtering and apply this technique to lane marking extraction in urban environment. Our method is based on two phases: an image preprocessing phase to extract pixels that potentially represent lanes and a tracking phase to identify lane markings. Compared to other approaches, our method presents a recursive filtering algorithm which extracts lane markings in the presence of clutter and non-lane markings.
\end{abstract}

The experimental results exhibit the high performance of the proposed approach under various scenarios.

\section{INTRODUCTION}

Within the past few years, active safety systems were increasingly introduced into cars to lower the number of accidents. The U.S. department of Transportation reported 33,963 fatalities in the year of 2009; 59\% of all traffic accidents were caused by lane departure. Therefore, road lane detection technology became a hot issue in the present intelligent vehicle research [1]. As a key part of advanced driver assistance systems, more and more researchers pay attention to lane detection research.

Hough transformation is the common method in the lane detection field to extract lines from images [2], [3], [4], [5]. However, the algorithms based on Hough transformation are typically only able to detect straight lanes.

Model based methods are also used to detect road lanes [6]. Zhou's method predefines a set of curvature models and selects the best fitting model per lane [7]. Although it can overcome the universal lane detection problems such as inaccuracies in edge detection caused by shadow of trees or passengers on the road, it is hard to combine these models. Edges in gray scale images are commonly considered as sufficient features for lane markings [8], [9], [10]. However, these features alone do not perform well in different light conditions or occlusions. Others predefine the road model and then use a Bayes filter to estimate the state [11], [12], [13], [14]. This method assumes that the lane has constant width on a flat plane and works well in these common cases. Limitations are in case of road lanes having more complicated shapes like in real traffic environments.

Feihu Zhang, Hauke Stähle, and Alois Knoll are with the Technische Universität München, Garching bei München, Germany, e-mail: feihu.zhang@tum.de, \{staehle,knoll\}@in.tum.de.

Christian Buckl and Chao Chen are with the fortiss $\mathrm{GmbH}$, München, email: \{buckl, chen\}@ fortiss.org.
As mentioned in [15], [16], [17], [18], the key issue is to extract the lane markings, which is crucial to the lane detection problem. In this paper, the focus is on road lane marking extraction by using a camera, gyroscope and GPS. We propose a method based on the Probability Hypothesis Density (PHD) filter [19]. In the PHD filter, the collection of individual targets is treated as set-valued states, while the collection of individual observations is treated as set-valued observations. Modeling set-valued states and set-valued observations as Random Finite Sets allows solving the problem of dynamically estimating multiple-targets in the presence of clutter and association uncertainty in a Bayesian filtering framework [20].

There are two phases in our approach: An image preprocessing phase and a tracking phase. In the first phase the image is cropped to extract the region of interest in the first step. In the next step, a median filter is utilized to reduce noise and retain the details. Then, Otsu's algorithm [21] is used to identify areas that potentially represent lane markings while image erosion is used to remove outliers. Finally, we transform those potential lane markings from image coordinates to vehicle coordinates (as defined in Fig. 3) with the goal of providing measurements.

The tracking phase is implemented in spatial dimension. In this stage the algorithm tracks lane markings by using the PHD filter.

The contribution of our approach is that it extracts the lane markings effectively from the complex urban environment. The influences from non-lane markings are eliminated since the PHD filter avoids the data association problem, where the whole potential lane markings are considered as the set-valued observation to update the set-valued state (the estimation of real lane markings).

Our approach is evaluated under real traffic scenarios. An off-the-shelf platform (iPhone4) was used to record data from the camera, gyroscope and GPS. The simulation illustrates that the proposed approach yields precise lane marking information in a complex urban environment.

The remainder of this paper is structured as follows: Sec. II briefly describes the image preprocessing stage. Sec. III introduces more details about the tracking phase. Sec. IV presents experimental results under traffic scenes. Finally, the paper is concluded in Sec.V.

\section{IMAGE PREPROCESSING PHASE}

The image preprocessing phase consists of two steps: the extraction step and the transformation step. 


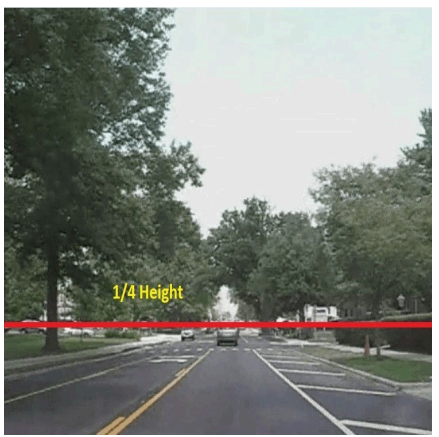

(a) Application of region of interest (ROI)

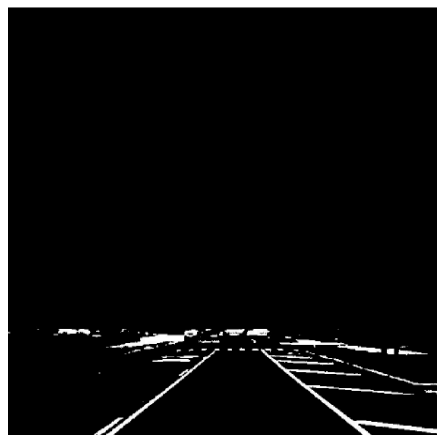

(b) Image after median filtering and application of Otsu's algorithm
Figure 1. Image preprocessing

In the extraction step potential pixels stemming from lane markings are extracted while in the transformation step the corresponding locations are transformed from image coordinates to vehicle coordinates.

The goal of the image preprocessing phase is to provide measurements (including the temporal and spatial information) for the tracking phase.

\section{A. Feature Extraction}

In most scenes, the road region and non-road region may have obvious boundaries. Furthermore, the road region is mainly under the horizon [22]. Consequently, we identify the road region of the image as the region of interest (ROI). This assumption also satisfies the efficiency and feasibility of the proposed approach. As shown in Fig. 1a, the image is divided vertically into two parts.

The image is then processed by a median filter to retain the details and remove the noise. Otsu's algorithm is used to calculate an adaptive threshold to segment the image into marking and non-marking areas [21]. Here the threshold is calculated in intensity rather than RGB. Fig. $1 \mathrm{~b}$ shows a simple segmentation result after the application of Otsu's algorithm.

After marking regions have been extracted, we apply image erosion to suppress outliers. The lane boundaries are extracted as follows: we start searching for lane pixels at the bottom row, left to right. After the current row is processed, we move on to the next row from bottom to top, line by line.

A pixel is identified as a potential lane pixel if it is part of a set of at least three horizontally connected pixels. The middlemost pixel of each set is recorded as a potential lane pixel. Fig. 2 illustrates the details of our method.

We also record the potential lane pixel's row index as the temporal information in the time domain for the tracking phase (Here the row index is calculated from the bottom row on the image and starts from 1).

\section{B. Coordinate Transformation}

We determine the mapping between the image coordinates $(u, v)$ of a tracked pixel and its corresponding point $(x, y)$ on the ground plane (vehicle coordinates). Fig. 3 shows that the

\begin{tabular}{|c|c|c|c|c|c|c|}
\hline$r / c$ & 1 & 2 & 3 & 4 & 5 & 6 \\
\hline 4 & 0 & 0 & 1 & 1 & 1 & 0 \\
\hline 3 & 0 & 1 & 1 & 1 & 1 & 0 \\
\hline 2 & 0 & 1 & 1 & 1 & 0 & 0 \\
\hline 1 & 1 & 1 & 1 & 0 & 0 & 0 \\
\hline
\end{tabular}

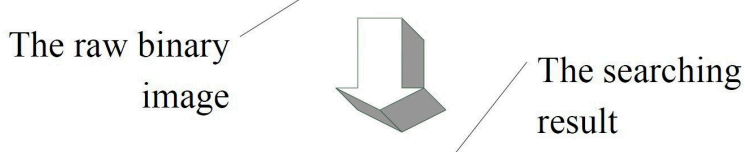

\begin{tabular}{|c|c|c|c|c|c|c|}
\hline $\mathrm{r} / \mathrm{c}$ & 1 & 2 & 3 & 4 & 5 & 6 \\
\hline 4 & 0 & 0 & 0 & 1 & 0 & 0 \\
\hline 3 & 0 & 0 & 1 & 0 & 0 & 0 \\
\hline 2 & 0 & 0 & 1 & 0 & 0 & 0 \\
\hline 1 & 0 & 1 & 0 & 0 & 0 & 0 \\
\hline
\end{tabular}

(a) The method used to identify lane pixels

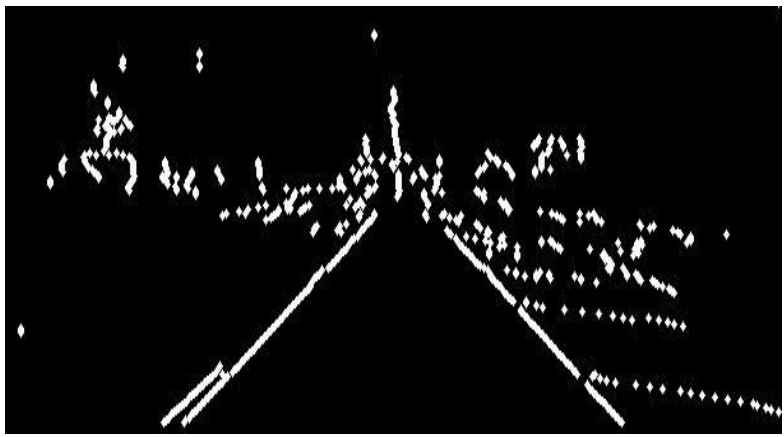

(b) Result of image preprocessing

Figure 2. Application of image erosion

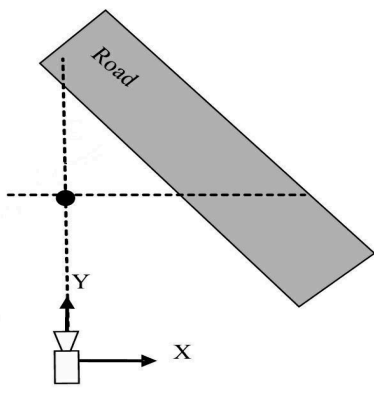

$\left[\begin{array}{lll}0 & 0 & 0\end{array}\right]^{\mathrm{T}}$

(a) Top view of the scene

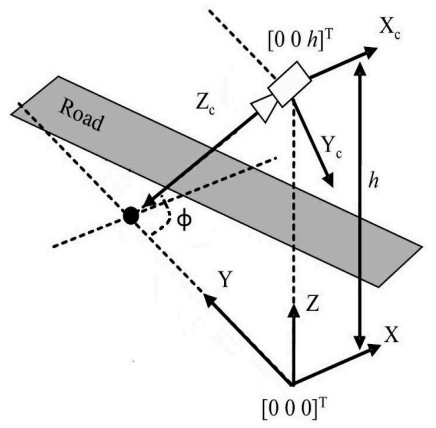

(b) Same scene viewed from the left side
Figure 3. Coordinate systems

camera is placed at height $h$ above the road with down/tilt angle $\phi . X, Y, Z$ are the axes of the vehicle's coordinate system while $X_{c}, Y_{c}, Z_{c}$, are the axes of the camera's coordinates system. Suppose the camera has zero skew, we transform the pixel's coordinates from image coordinates to vehicle coordinates as follows [23]:

A point $\mathbf{P}=[x, y, z, 1]^{T}$ in the vehicle coordinates is re- 
lated to its image coordinates $\mathbf{p}=[u w, v w, w]^{T}$,

$$
\begin{gathered}
{\left[\begin{array}{c}
u w \\
v w \\
w
\end{array}\right]=K R\left[I_{3 \times 3} \mid-T\right]\left[\begin{array}{l}
x \\
y \\
z \\
1
\end{array}\right]} \\
K=\left[\begin{array}{ccc}
f & 0 & u_{c} \\
0 & f & v_{c} \\
0 & 0 & 1
\end{array}\right] \\
R=\left[\begin{array}{ccc}
1 & 0 & 0 \\
0 & -\sin \phi & -\cos \phi \\
0 & \cos \phi & -\sin \phi
\end{array}\right]
\end{gathered}
$$

where $K$ is the camera calibration matrix, $R$ is the rotation matrix corresponding to a rotation of $\phi$ around the X-axis, $I$ is the identity matrix and $T=[0,0, h]^{T}$ is the translation of the camera from the origin of the vehicle coordinate system. $\left[I_{3 \times 3} \mid-T\right]$ is the concatenation of $I$ and $T .\left(u_{c}, v_{c}\right)$ are the principal point coordinates. The actual pixel coordinates $(u, v)$ are defined with respect to the origin in the top left hand corner of the image plane and $w$ is a scale factor.

Using equation (1) we can express the relationship between the vehicle coordinates $\left(x_{n}, y_{n}, z_{n}\right)$ of a point on the road $\left(z_{n}=0\right)$ to its image coordinates $\left(u_{n}, v_{n}\right)$ at frame $n$ as follows:

$$
\begin{gathered}
u_{n}=\frac{w u_{n}}{w}=\frac{f x_{n}}{y_{n} \cos \phi+h \sin \phi}+u_{c} \\
v_{n}=\frac{w v_{n}}{w}=\frac{f h \cos \phi-f y_{n} \sin \phi}{y_{n} \cos \phi+h \sin \phi}+v_{c}
\end{gathered}
$$

Rearranging the above equations, we get

$$
\begin{gathered}
x_{n}=\frac{\left(u_{n}-u_{c}\right)(h f \cos \phi+f h \tan \phi \sin \phi)+2 h u_{c} \sin \phi\left(v_{c}-v_{n}\right)}{f\left(v_{n}+f \tan \phi-v_{c}\right)} \\
y_{n}=\frac{h\left(f-v_{n} \tan \phi+v_{c} \tan \phi\right)}{v_{n}+f \tan \phi-v_{c}}
\end{gathered}
$$

\section{LANE TRACKING PHASE}

This phase is implemented in vehicle coordinates. We classify the potential lane pixels as clutter or lane markings. Knowing that a road lane can be represented as a lane marking's integrity track, the tracking phase is proposed to track the real lane markings.

Since each pixel's row value is used as the time index (ref. Sec. II-A) and the corresponding positions are available (ref. Sec. II-B), a tracking method based on RFS is established to distinguish between clutter and lane pixels.

\section{A. Overview on RFS Statistics}

The Random Finite Set (RFS) is a hidden markov chain model with set-valued states and set-valued observations while the PHD filter is a predict and correct framework for recursive Bayesian filtering in such a RFS formulation. A comparison of the RFS approach and traditional multiple-target tracking methods has been given in [24]. In the PHD filter, the collection of individual targets is treated as set-valued states,

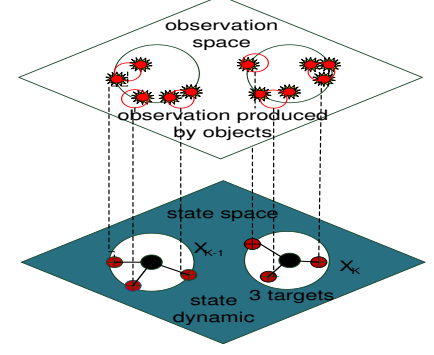

Figure 4. Set-valued states and set-valued observations

and the collection of individual observations is treated as set-valued observations. Fig. 4 is a basic introduction of the PHD filter which shows that the observations and their estimated states are treated as a single valued measurement and its corresponding estimation at each frame [25]. The PHD filter operates on the single-target state space and avoids the combinatorial problem that arises from data association.

Our previous work also considered the PHD filter as a solution to visual odometry [26], [27]. The proposed approach estimated the ego-motion of the vehicle without concerning the association issues of the corresponding features.

H. Deusch et. al. [28] implemented the PHD filter in the field of lane detection. They focus on the dependencies between multiple lanes without explicit data association in post processing. In this paper, we use the PHD filter to extract the lane markings from the potential lane pixels. Compared to Deusch's approach, our approach works in the preprocessing phase in the lane detection field (lane marking extraction).

\section{B. Mathematic Background on the PHD Filter}

The PHD filter is an approximation to alleviate the computational intractability of the optimal multi-target Bayes filter, proposed by Mahler [19]. Considering the RFS of survived targets $S_{k \mid k-1}$ between iterations $k-1$ and $k$, the RFS of spawned targets $B_{k \mid k-1}$ and the RFS of spontaneous birth targets $\sigma_{k}$, the global RFS characterizing the multiple-target set can be written as:

$$
\mathbf{X}_{k}=\left[\bigcup_{\zeta \in \mathbf{X}_{k-1}} S_{k \mid k-1}(\zeta)\right] \cup\left[\bigcup_{\zeta \in \mathbf{X}_{k-1}} B_{k \mid k-1}(\zeta)\right] \cup \sigma_{k}
$$

The set observation $\mathbf{Z}_{k}$ can be seen as a global RFS composed by the RFS of measurements originally from the targets $\theta_{k}(\mathbf{x})$ and by the RFS of false alarms $\kappa_{k}$, modeled by a Poisson distribution.

$$
\mathbf{Z}_{k}=\left[\bigcup_{\mathbf{x} \in \mathbf{X}_{k}} \theta_{k}(\mathbf{x})\right] \cup \kappa_{k}
$$

The PHD filter evolves in two steps: prediction and update. The multiple-target posterior density of the target RFS is also called the intensity function $D$. The transition function $f_{k \mid k-1}(\mathbf{x} \mid \zeta)$ given the previous state $\zeta$. 
The prediction equation of the PHD filter is:

$D_{k \mid k-1}(\mathbf{x})=\left[\int\left[P_{S}(\zeta) f_{k \mid k-1}(\mathbf{x} \mid \zeta)+\beta(\mathbf{x} \mid \zeta)\right] D_{k-1}(\zeta) d \zeta\right]+\gamma_{k}$

Knowing the measurement random set $\mathbf{Z}_{k}$, it is possible to update the intensity function as follows:

$$
\begin{gathered}
D_{k}(\mathbf{x})=\left(1-P_{D}\right) D_{k \mid k-1}(\mathbf{x}) \\
+\sum_{z \in \mathbf{Z}_{k}} \frac{P_{D} g_{k}\left(\mathbf{z}_{\mathbf{i}} \mid \mathbf{x}\right) D_{k \mid k-1}(\mathbf{x})}{\kappa\left(\mathbf{z}_{\mathbf{i}}\right)+\int P_{D} g_{k}\left(\mathbf{z}_{\mathbf{i}} \mid \zeta\right) D_{k \mid k-1}(\zeta) d \zeta}
\end{gathered}
$$

where $P_{D}$ is the probability of detection. $g_{k}\left(\mathbf{z}_{\mathbf{i}} \mid \mathbf{x}\right)$ is the likelihood of state $\mathbf{x}$ given an observation $\mathbf{z}_{\mathbf{i}}$.

The PHD prediction equation (8) includes components whose intensities are affected by targets that enter the scene $\left(\gamma_{k}\right)$, targets that spawn new targets $(\beta(\mathbf{x} \mid \zeta))$, and targets that survive from the previous time step $P_{S} \cdot D_{k-1}$ is the posterior PHD from the previous time step.

The PHD update equation (9) corrects the predicted PHD by including evidence from the current set of observations. Knowledge about scene clutter $\kappa(\mathbf{z})$ is also embedded into the update step. $\kappa(\mathbf{z})$ is the intensity of clutter (expected number of observations arising from the clutter at $\mathbf{z}$ ).

$$
N(k)=\int_{\Psi} D_{k \mid k}(\mathbf{x}) d \mathbf{x}
$$

Equation (III-B) illustrates that the integral of the PHD over a certain domain $\Psi$ yields the estimated number of targets $N(k)$ in that domain at time $k$. The PHD is not a probability density and does not necessarily sum up to 1[19].

It is to be noted that the PHD recursion involving equations (8) and (9) have multiple integrals that have no closed form solutions in general. One of the common approaches to mitigate this problem is to use GM-PHD (Gaussian Mixture) approximations. More details and a generic description of the GM-PHD filter is given in [29].

\section{Implementation Details}

1) Motion Model and Observation Model: Similar to the standard target tracking approaches, we consider the lane pixel's motion and observation process as follows:

$$
\begin{aligned}
\mathbf{x}_{k+1} & =\mathbf{F}_{k} \mathbf{x}_{k}+\mathbf{w}_{k} \\
\mathbf{z}_{k+1} & =\mathbf{H}_{k+1} \mathbf{x}_{k+1}+\mathbf{v}_{k+1}
\end{aligned}
$$

where the time index $k$ and the state vector $\mathbf{x}_{k}=$ $\left(x_{k}, y_{k}, \dot{x}_{k}, \dot{y}_{k}\right)^{T}$ describe the position $\left(x_{k}, y_{k}\right)$ and velocity $\left(\dot{x}_{k}, \dot{y}_{k}\right)$ in vehicle coordinates; $\mathbf{F}$ is the state transition matrix. The process noise $\mathbf{w}_{k}$ is described by a white zero mean, normal distributed random process with covariance $\mathbf{Q}$.

$$
\mathbf{F}=\left[\begin{array}{cccc}
1 & 0 & T & 0 \\
0 & 1 & 0 & T \\
0 & 0 & 1 & 0 \\
0 & 0 & 0 & 1
\end{array}\right]
$$

where $T$ denotes a discrete sampling period. In the case of our algorithm, the sample period reflects the steps of row-wise calculation in Sec. II-A.
The measurement is given by the potential lane pixel's coordinates and is modeled with equation (11), where $\mathbf{H}$ is the observation matrix and $\mathbf{v}_{k}$ refers to the white zero mean, normal distributed measurement noise with covariance $\mathbf{R}$.

To map the state vectors to the observation space, the observation matrix is:

$$
\mathbf{H}=\left[\begin{array}{llll}
1 & 0 & 0 & 0 \\
0 & 1 & 0 & 0
\end{array}\right]
$$

2) Pruning and Merging: Aggregated lane pixels falling below a given threshold are pruned and the remaining pixels are reweighed accordingly. If the distance of the state defined by the covariance matrix falls within a merging threshold $\tau$, then the lane pixels are merged.

3) Birth Model: The birth model depends on the observations in a certain region. In this paper we consider the distance between the lanes (left lane and right lane) is a constant value $(3 \mathrm{~m})$. Assuming that the distance between the vehicle and the right lane is $\sigma_{n}$ at frame $n$, lane pixels will be born at the places $\left[\sigma_{n}-0.1, \sigma_{n}+0.1\right]$ and $\left[\sigma_{n}-0.1-3, \sigma_{n}+0.1-3\right]$ in $X$ direction in vehicle coordinates (here 0.1 is the threshold parameter for the birth model). For instance, if the vehicle is in the middle of the lanes, the birth region will be $(1.4,1.6)$ for the right lane and $(-1.6,-1.4)$ for the left lane. In addition, the birth pixels are only established within a range of a few meters in $Y$ direction, which is beneficial for the clutter removing.

A dead reckoning method is used to calculate the parameter $\sigma_{n}$ by fusing the data from the GPS and gyroscope sensors in consecutive frames [30]. The GPS provides the velocity $v_{n}$ of the vehicle and the gyroscope sensor provides the vehicle's angular change $\beta_{n}$ between consecutive frames.

$$
\sigma_{n}=\sum_{i=1}^{n} v_{n} \sin \beta_{n}
$$

According to this assumption, the measurements which enter certain regions can be used to initialize the birth models. If no birth occurred in $2 s$, the distance $\sigma_{n}$ will be reinitialized at the middle of the road lanes (here $1.5 \mathrm{~m}$ ).

4) Spawn Model: In our case there is no lane pixel spawn from the existed lane pixels.

We use the PHD filter to track the lane pixels at each individual frame. The only related parameters between consecutive frames is the distance $\sigma_{n}$, which is used for generalizing the birth models.

\section{EXPERIMENTAL RESULTS}

The lane detection algorithm described in this paper has been implemented on a Core 2 Duo 3.0Ghz computer. The algorithm is executed in Matlab and the average processing time in the image preprocessing phase is $70 \mathrm{~ms}$ per frame and $15 \mathrm{~ms}$ per frame in tracking phase. An iPhone4 platform is used to provide data including GPS, gyro and images at 30 frames/s with a resolution of $480 \times 640$ pixels. The sequences represent real traffic conditions in urban environments with shadows and other cars. In the experiments, the vehicle was driven with a constant velocity of $50 \mathrm{~km} / \mathrm{h}$. 
Table I

PERFORMANCE OF THE ALGORITHM

\begin{tabular}{l|l|l}
\hline Video & DR & FR \\
\hline Urban Road & $93.6 \%$ & $6.2 \%$ \\
\hline
\end{tabular}

Fig. 5 exhibits the results of our approach on curvy lanes in the city. Fig. 5a is the original image acquired from the camera. Fig. $5 b$ shows the result of the image preprocessing phase while Fig. $5 \mathrm{c}$ illustrates the PHD filter results in tracking phase. The real lane markings are extracted effectively in the highly cluttered environment. Fig. 5d draws the corresponding lane markings on the original image. The reason why the lane markings near the right lane are not well extracted is due to the fact that the threshold value calculated by Otsu's algorithm is too high. Nevertheless, it is not the fault of the PHD filter. In future work, we will consider alternative threshold approaches to investigate the performance of the proposed approach.

Fig. 6 shows the result of our approach on straight lanes. From Fig. 6c and Fig. 6d we can see that our approach can also extract the lane markings on the attached lanes. This is due to the measurements which fall in the birth regions that were calculated by fusing the sensors.

The performance of our approach is also shown in table I. DR denotes the successful lane marking extraction rate while FR is the false rate. Lane pixels on the ground are manually marked and recorded in a database. The database includes image sequences with the corresponding data acquired in Munich under various scenarios. The criterion of successful extraction is based on the distances between real lane pixels and the PHD filter's results in one image.

$$
D R=\frac{N T P}{N G L}, F R=1-\frac{N T P}{N D R}
$$

$N G L$ represents the number of real lane pixels in the evaluation set. $N T P$ is the number of lane pixels which are extracted successfully. $N D R$ is the number of extracted pixels by the PHD filter.

The results of our experiments indicate that the PHD filter performs robustly for lane marking extraction in the presence of clutter and non-markings.

Compared to others, the benefits of our approach are concluded as follows:

1) The idea of replacing the vertical image with the time domain is derived in this paper, which transforms the lane marking extraction problem to the standard target tracking problem.

2) The PHD filter extracts the lane markings effectively in the urban environment. It is difficult to remove nonlane markings in the image, which may influence the precision of the lane detection. However, within the RFS framework, the PHD filter avoids the association issues. The pixels are considered together as set-valued observations to update the set-valued state (real lane markings).

3) The PHD filter not only extracts the lane markings from straight lanes, but also from curved lanes. Both, Hough transformation methods and model based methods, need a road lane model to be predefined. However, the PHD filter avoids this issue by assuming that the road lane can be represented as a lane marking's integrity track, which transforms the shape estimation problem to a point estimation problem.

These salient features render the PHD filter very promising to the lane detection field.

\section{CONCLUSION}

It is very hard to extract road lane markings in urban environments. The varying road signs and clutter from other objects render the extraction process difficult. In this paper, an approach of PHD filtering under the RFS framework is presented. In comparison to earlier works, this paper is among the first to apply PHD filtering to lane marking extraction in real traffic scenes. The proposed approach not only extracts the real lane markings in the presence of clutter and non-lane markings, but also avoids the dependency from the shapes of the lanes. The evaluation results indicate that the algorithm achieves high accuracy and robustness under different scenes.

Future improvements may include visual odometry techniques to improve the estimation precision and robustness.

\section{REFERENCES}

[1] X. Dai, A. Kummert, S. B. Park, and D. Neisius, "A warning algorithm for lane departure warning system," in Intelligent Vehicles Symposium, 2009 IEEE, 2009, pp. $431-435$.

[2] A. Saudi, J. Teo, M. H. A. Hijazi, and J. Sulaiman, "Fast lane detection with randomized hough transform," in Information Technology, 2008. ITSim 2008. International Symposium on, vol. 4, 2008, pp. 1 -5.

[3] J. McCall and M. Trivedi, "An integrated, robust approach to lane marking detection and lane tracking," in Intelligent Vehicles Symposium, 2004 IEEE, june 2004, pp. 533 - 537.

[4] A. Assidiq, O. Khalifa, R. Islam, and S. Khan, "Real time lane detection for autonomous vehicles," in Computer and Communication Engineering, 2008. ICCCE 2008. International Conference on, may 2008, pp. $82-88$.

[5] G. Liu, F. Worgotter, and I. Markelic, "Combining statistical hough transform and particle filter for robust lane detection and tracking," in Intelligent Vehicles Symposium (IV), 2010 IEEE, june 2010, pp. 993 -997 .

[6] K. Zhao, M. Meuter, C. Nunn, D. Muller, S. Muller-Schneiders, and J. Pauli, "A novel multi-lane detection and tracking system," in Intelligent Vehicles Symposium (IV), 2012 IEEE, june 2012, pp. 1084 -1089.

[7] S. Zhou, Y. Jiang, J. Xi, J. Gong, G. Xiong, and H. Chen, "A novel lane detection based on geometrical model and gabor filter," in Intelligent Vehicles Symposium (IV), 2010 IEEE, 2010, pp. 59 -64.

[8] K. Kluge and S. Lakshmanan, "A deformable-template approach to lane detection," in Intelligent Vehicles '95 Symposium., Proceedings of the, sep 1995, pp. $54-59$.

[9] F.Paetzold and U.Franke, "Road recognition in urban environment," in Image and Vision Computing, vol. 18, 2000, pp. 377 -387.

[10] Y.Wang, E.Teoh, and D.Shen, "Lane detection and tracking using bsnake," in Image and Vision Computing, vol. 18, April 2004, pp. 269 -280 .

[11] A. Takahashi and Y. Ninomiya, "Model-based lane recognition," in Intelligent Vehicles Symposium, 1996., Proceedings of the 1996 IEEE, Sept. 1996, pp. $201-206$.

[12] M. Bertozzi and A. Broggi, "Gold: a parallel real-time stereo vision system for generic obstacle and lane detection," Image Processing, IEEE Transactions on, vol. 7, no. 1, pp. 62 -81, Jan. 1998.

[13] P. Coulombeau and C. Laurgeau, "Vehicle yaw, pitch, roll and 3d lane shape recovery by vision," in Intelligent Vehicle Symposium, 2002. IEEE, vol. 2, june 2002, pp. $619-625$ vol.2. 


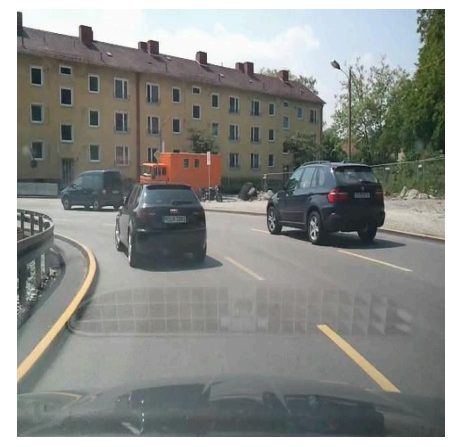

(a)

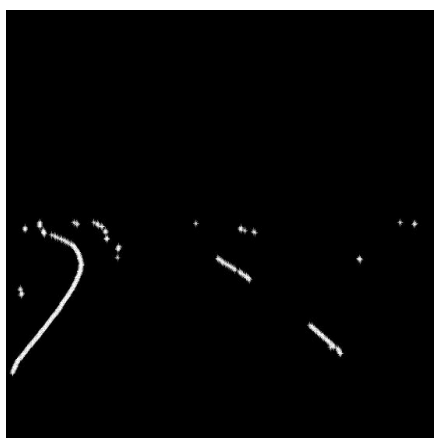

(b)

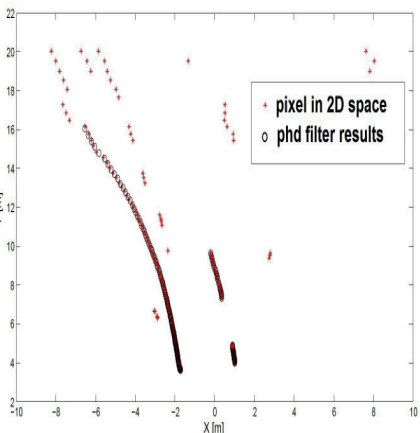

(c)

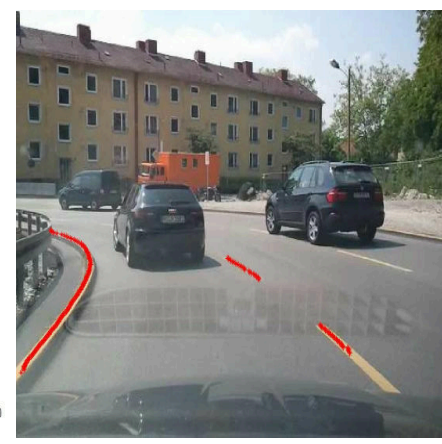

(d)

Figure 5. Lane detection on curvy lanes

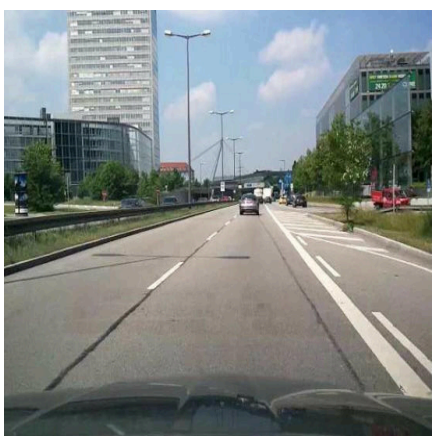

(a)

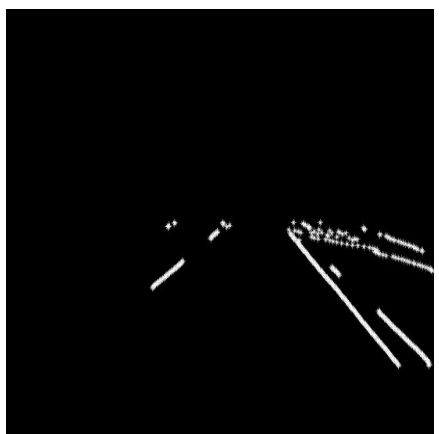

(b)

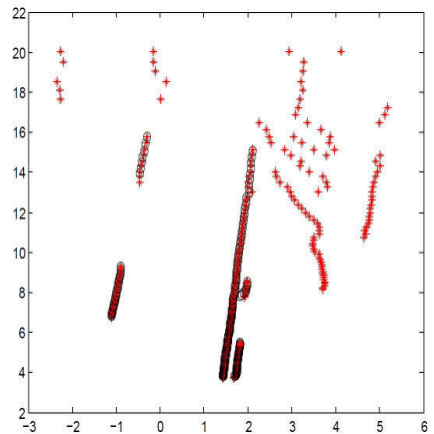

(c)

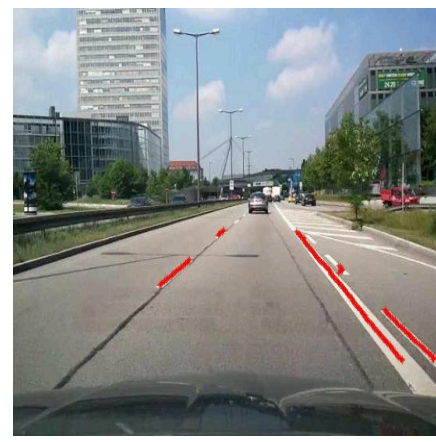

(d)

Figure 6. Lane detection on straight lanes

[14] Z. Kim, "Robust lane detection and tracking in challenging scenarios," Intelligent Transportation Systems, IEEE Transactions on, vol. 9, no. 1, pp. $16-26,2008$.

[15] T. Veit, J.-P. Tarel, P. Nicolle, and P. Charbonnier, "Evaluation of road marking feature extraction," in Intelligent Transportation Systems, 2008. ITSC 2008. 11th International IEEE Conference on, oct. 2008, pp. 174 -181 .

[16] E. Pollard, D. Gruyer, J.-P. Tarel, S.-S. Ieng, and A. Cord, "Lane marking extraction with combination strategy and comparative evaluation on synthetic and camera images," in Intelligent Transportation Systems (ITSC), 2011 14th International IEEE Conference on, oct. 2011, pp. $1741-1746$.

[17] R. Satzoda, S. Suchitra, and T. Srikanthan, "Robust extraction of lane markings using gradient angle histograms and directional signed edges," in Intelligent Vehicles Symposium (IV), 2012 IEEE, june 2012, pp. 754 -759 .

[18] Y. Sebsadji, J.-P. Tarel, P. Foucher, and P. Charbonnier, "Robust road marking extraction in urban environments using stereo images," in Intelligent Vehicles Symposium (IV), 2010 IEEE, june 2010, pp. 394 -400 .

[19] R. Mahler, "Multitarget bayes filtering via first-order multitarget moments," IEEE Transactions on Aerospace and Electronic Systems, no. 4, pp. 1152 - 1178, Vol. 39, Oct. 2003.

[20] B.-N. Vo, S. Singh, and A. Doucet, "Sequential monte carlo implementation of the phd filter for multi-target tracking," in Proceedings of the Sixth International Conference on Information Fusion, 2003, pp. 792 799, Vol. 2.

[21] N. Otsu, "A threshold selection method from gray-level histograms," Systems, Man and Cybernetics, IEEE Transactions on, vol. 9, no. 1, pp. $62-66$, jan. 1979.

[22] W.N.Lu and Z.K.Shi, "Synchronous detection of the lane marking and road boundary on monocular vision," Chinese Journal of Sensors and Actuators, vol. 20, pp. 1171 - 1175, May 2007.

[23] R. Hartley and A. Zisserman, Multiple View Geometry in Computer
Vision. Cambridge University Press, 2000.

[24] I. Goodman, R. Mahler, and H. Nguyen, Mathematics of Data Fusion. Norwell, MA: Kluwer Academic, 1997.

[25] B.-N. Vo, "Random finite sets in stochastic filtering," EEE Department University of Melbourne Australia, Tech. Rep., 2009. [Online]. Available: http://www.ee.unimelb.edu.au/staff/bv/

[26] F. Zhang, G. Chen, H. Stahle, C. Buckl, and A. Knoll, "Visual odometry based on random finite set statistics in urban environment," in Intelligent Vehicles Symposium (IV), 2012 IEEE, june 2012, pp. 69 -74.

[27] F. Zhang, H. Stahle, A. Gaschler, C. Buckl, and A. Knoll, "Single camera visual odometry based on random finite set statistics," in Intelligent Robots and Systems (IROS), 2012 IEEE/RSJ International Conference on, 2012, pp. 559-566.

[28] H. Deusch, J. Wiest, S. Reuter, M. Szczot, M. Konrad, and K. Dietmayer, "A random finite set approach to multiple lane detection," in Intelligent Transportation Systems (ITSC), 2012 15th International IEEE Conference on, sept. 2012, pp. $270-275$.

[29] B.-N. Vo and W.-K. Ma, "The gaussian mixture probability hypothesis density filter,' IEEE Transactions on Signal Processing, no. 11, pp. 4091 -4104, Vol. 54, Nov. 2006.

[30] K. Park, H. Chung, J. Choi, and J. G. Lee, "Dead reckoning navigation for an autonomous mobile robot using a differential encoder and a gyroscope," in Advanced Robotics, 1997. ICAR '97. Proceedings., 8th International Conference on, jul 1997, pp. $441-446$. 